\title{
Determination of time dependent crack contact behaviour by thermoelastic stress analysis
}

\author{
by O. Schlicht and H.-P. Wölfel
}

Technical University of Damstadt, D-64287 Darmstadt, Germany

\begin{abstract}
Using the Thermoelastic Stress Analysis the time history of the sum of principal stresses (SPS) along the faces of through-thickness cracks in sinusoidally loaded plates is examined. The influence of crack closure on the local stresses is shown and thus the exact moment of local crack opening and closure as well as the local crack opening load are determined. The effect of static preloads on the plate - due to remote loading or residual stresses - is also monitored. Differences between the contact behaviour of cracks in plates made of linear-elastic or elastic-plastic material are shown.
\end{abstract}

\section{Introduction}

\subsection{Thermoelastic stress analysis}

The Thermoelastic Stress Analysis (THESA) is a noncontacting technique for the experimental determination of the sum of principal stresses (SPS) on the surface of dynamically loaded structures by the measurement of infrared emission. The signal measured depends on reversible energy conversion on the surface of the loaded specimen and not on dissipated energy like in other thermal measuring methods such as Vibrothermography. THESA is based on the thermoelastic effect. For a linear-elastic, isortropic solid subjected to adiabatic, isentropic loading conditions the effect is described by the basic equation

$$
\Delta T=-K_{m} T_{0} \Delta \sigma_{1}
$$

with $\Delta T=$ change of temperature; $K_{m}=$ thermoelastic constant; $T_{0}=$ mean surface temperature; $\Delta \sigma_{1}=$ change of SPS. The basic equation (1) presumes that adiabatic conditions prevail. In order to ensure these conditions the specimen has to be loaded cyclically. Then the minute temperature changes on the surface of the structure can be measured with the system SPATE [1] that has been available since the early 1980's. Due to eq.(1) constant stresses usually cannot be measured. Each measurement determines the temperature change and thus the change of the SPS at one single point. By the automatic joining of several measurements linear and areal measurements are also possible.

In order to reduce the noise in the measurement signal from the infrared camera, the signal is usually correlated by an electronic data processing system with a reference signal from the loading system. Then as the result of the measurement at each point the first or second Fourier's coefficient is given. A detailed description of the technique and a survey of possible uses is given in [2]. In the area of fracture mechanics different ranges of application are known. The detection of unknown cracks by different methods is described in [3] and [4]. In [4] and in [5] the geometry of semielliptical surface cracks is determined in order to estimate the significance of the damage. The determination of the important stress intensity factor by a specific procedure is described in [6]. All methods described above use the first and/or second Fourier's coefficient of the measurement signal.

\subsection{Fracture mechanics}

An important aspect in the investigation of dynamically loaded cracks is the contact of the crack faces. If a plate with a through-thickness crack is loaded by a sinusoidal bending force (fig.1), then the crack faces open and close alternately. On the compressive surface of the plate the crack is closed and does not undergo any harmful stress. On the tensile surface of 
the plate the crack is open and crack growth is possible. As the plate is loaded sinusoidally crack contact occurs on the top and on the bottom surface of the plate alternately. Thus all important effects occur on both sides. The present examples all refer to the top surface of the plate, where in the case of linear elastic material behaviour the crack is either totally open along its entire length of $2 a$ or it is totally closed.

In the case of elastic-plastic material behaviour the contact mechanism is different. Elber [7] was the first to mention that due to elastic-plastic material behaviour residual compressive stresses exist between the faces of a crack in a dynamically loaded structure. The magnitude of these residual stresses depends on the distance from the crack tip. The crack is only open if the remote tensile loading exceeds the residual stresses. Hence crack opening and closure depends on the location along the crack face and does not occur simultaneously along the entire crack length. As the crack tip only advances if the crack is completely open, the determination of crack opening and closure loads has become an important task. Several techniques for the experimental investigation of crack tip opening are known. Most of them use strain gauges, thus providing information about specimen strain, which is then analyzed to determine the crack opening load [8].

The SPATE system monitors the stress state on the surface of the structure and the crack opening behaviour directly. Since the infrared system needs less preparatory effort than the methods mentioned above (merely aiming the infrared detector at a different point instead of applying a new strain gauge), flexibility is increased especially when propagating cracks are investigated. However, it must be taken into consideration that in the close vicinity of the crack tip elastic-plastic material behaviour prevails. Further investigations about the applicability of the technique in that region are necessary before quantitative measurements exactly at the crack tip can be carried out.

\section{Measurement technique}

In order to test the applicability of the Thermoelastic Stress Analysis for the investigation of crack opening, plates with linear-elastic and elastic-plastic material behaviour are used. Each measurement is conducted at one single point selected at the crack face. In order to realize a detailed investigation of the contact behaviour, the results have to be displayed in the time domain. This is in contrast to usual processing where the results are displayed in the frequency domain. Due to the considerable noise in the signal from the infrared camera, additional steps have to be taken to allow for an analyzation of the results.

The signal is first low-pass filtered and then averaged in the time domain using the loading signal as trigger. Thus, most of the high frequency noise is eliminated and the results can be displayed as time history.

\section{Contact in case of linear elastic material behaviour}

The studies based on linear-elastic material behaviour are performed on plates made of epoxy resin with artificially produced cracks (figure 1). During the casting of the plates a small strip of teflon is placed in the plate. On one side the strip is coated with parting compound so that the epoxy resin comes into contact with that side of the strip but does not stick on it. Consequently only compressive forces and no tensile forces can be transferred by the crack faces.

\subsection{Calculations}

In order to simulate the stress distribution at the crack faces, calculations with the FiniteElement program ABAQUS [9] are carried out. In this study the results are analyzed at point $A$ on the top side of the plate (figure 1). Figure 2 shows the time history of the SPS as result of a calculation in case of sinusoidal loading. Significant bends in the time history of the SPS indicate the exact moments of crack opening and closure. If the remote load is negative the crack closes. The crack faces get into contact and transfer compressive stresses. If the load is 
positive (direction of the force in figure 1) the crack opens. As the lateral deformation along the crack faces is hindered, the stress component $\sigma_{x}$ is negative und thus the SPS at point A is also negative during crack opening and not zero as might be expected. Obviously the time history of the SPS is no longer sinusoidal, because the changes in contact at the crack produce a geometric nonlinearity within the structure. Loading the nonlinear system sinusoidally leads to a periodic stress time history. Its Fourier series does not only consist of the excitation frequency itself but also of multiples of the excitation frequency and of a constant portion.

In order to compare the results of the calculation with the results of the measurements it has to be considered that when carrying out a THESA-measurement only time depending portions of the stresses in the structure can be detected. Whereas the higher frequencies are still in the measurement signal, the constant portion is missing. Hence the curve is shifted vertically so that

$$
\sigma_{1}=\sigma_{1}^{m}+\text { const }
$$

with $\sigma_{1}=$ SPS on the structure, $\sigma_{1}^{m}=$ SPS as it is measured with SPATE. The shift is shown by the dotted line in fig. 2 which displays the calculated SPS without a constant portion. As the constant portion is missing no absolute values from the time history can be used. Nevertheless differences between values at different instants can be calculated.

\subsection{Measurements}

In figure 3 the results of a measurement accomplished in analogy to the calculations are shown. The plate is sinusoidally loaded with an excitation frequency of $20 \mathrm{~Hz}$ which is clearly below the first eigenfrequency of the plate. In the time history the bends caused by crack opening and closure can be clearly recognized. If the crack is open the influence of the hindered lateral deformation seems to be small. As explained above the missing of the constant portion causes a vertical shift in the time history. The vertical auxiliary lines verify that the crack on the upper surface is open at positive loads and closed at negative loads (direction of the force in figure 1). This is in good agreement with the calculations.

During the next tests a constant portion is added to the sinuoidal portion of the load. The load time history is shown in figures 4 and 5 . Although the constant portion cannot be measured directly with SPATE it has a significant influence on the contact behaviour of the crack and also on the time history of the measured SPS at the crack faces: If the static load is tensile (positive force) the crack at the point of investigation is much longer open than in the case without static load. The curves in figure 4 show that the crack is open if the sum of both loads (constant load and sinusoidal load) is positive.

The load case of a superimposed static compressive load (negative force) is more interesting because it is quite similar to the case of compressive residual stresses that will be discussed below. Again the crack opens if the sum of both loads (constant and sinusoidal load). is positive (figure 5). But here the crack is much longer closed than in both cases mentioned above.

Even if the static portion of the load $L_{s t}$ is unknown and only the sinusoidal portion can be recorded (dotted curve in figure 5 ), the local crack opening load $L_{\text {opp }}$ i.e. the remote load at which the crack opens locally, can be determined. As shown in fig.5 it results from the intersection point of the load curve with the vertical auxiliary line that marks the crack opening. In that example the crack opens at $L_{\text {op }}=20 \mathrm{~N}$.

The results show that the contact behaviour can be studied at selected points along the crack faces. By analyzing the measured time histories of load and SPS it is possible to determine the local crack opening load. 


\section{Contact in case of elastic-plastic material behaviour}

The investigations concerning the influence of elastic-plastic material behaviour are conducted at a through-thickness crack initiated by a notch in a plate of Al5086. The plate is subjected to bending by a sinusoidal force of $20 \mathrm{~Hz}$ which is clearly below the first eigenfrequency of the plate. The measurements are taken at three different points (figure 6). During the measurements all three points lie outside the plastically deformed area. As the present tests are conducted in order to determine static residual stresses in the plate, no remote static loads must be applied. Otherwise both static portions could not be separated.

At point 1 sufficiently far away from the crack the time history of the SPS $\sigma_{1}(t)$ is sinusoidal and equal to that of an uncracked plate. The maximum of the SPS measured is $\sigma_{1}^{\mathrm{m}}=60 \mathrm{MPa}$.

At point 2 beside the crack the moment of crack opening can be clearly determined by bends in the time history (figure 7). The instant when the load becomes positive and the instant of crack opening are shown by two vertical auxiliary lines. It is noticeable that the crack does not open when the remote load becomes positive - as it has been observed in the case of linear-elastic material behaviour. It opens at a tensile applied load. This is explained by the influence of elastic-plastic material behaviour. Caused by yielding at the crack tip during crack growth there exists residual compressive stress at the crack faces. If the crack starts opening

$$
\sigma_{\text {bad }}\left(t_{\text {open }}\right)-\sigma_{\text {res }}=0
$$

with $\sigma_{\text {load }}=$ stress in y-direction due to remote loading; $\sigma_{\text {res }}=$ residual stress in y-direction; $t_{\text {open }}=$ instant when the crack starts opening. This is similar to the case mentioned above, where the crack was only open if the sinusoidal load has exceeded the static compressive load. Here the crack opens at $L_{\text {op }}=53 \mathrm{~N}$.

The influence of constant residual stress on the measured signal can be shown, because it is possible to determine when the crack faces at the measurement point separate. Assuming uniaxial stress state along the crack faces and considering that the time-history of $\sigma_{1}^{m}$ is shifted so that only differences can be used for further analyzation, it yields

$$
\sigma_{\text {bad }}\left(\mathrm{t}_{\text {open }}\right)-\sigma_{\text {bad }}\left(\mathrm{t}_{0}\right) \approx \sigma_{1}^{\mathrm{m}}\left(\mathrm{t}_{\text {open }}\right)-\sigma_{1}^{\mathrm{m}}\left(\mathrm{t}_{0}\right)
$$

with $t_{0}=$ instant when the remote load equals zero; $t_{\text {open }}=$ instant when the crack opens. As there is no remote load at to the stress due to that load also equals zero:

$$
\sigma_{\text {foad }}\left(t_{0}\right)=0
$$

Then with eqs.(3) and (4) the residual stresses can be estimated by the data of fig.7

$$
\sigma_{\text {res }}=-\sigma_{\text {load }}\left(\mathrm{t}_{\text {open }}\right) \approx-24 \mathrm{MPa}
$$

As explained above due to compressive residual stress also the crack faces at point 3 , that is closer to the crack tip. (measurement taken at a different plate) separate at a tensile load stress (figure 8). Crack closure though occurs at a significantly lower stress. This difference can be explained by yielding at the crack tip where the stress is increased extremely after crack opening. This plastic deformation reduces the residual stress close to the crack tip so that the crack closes at lower load stress. Under compression the plastic strain is reduced so that the sequence is repeated.

\section{Conclusions}

The present examples show that the THESA is a flexible tool to monitor the time history of the SPS. The moment of local crack opening and closure can be detected. The influence of 
static loads is also revealed. In case of elastic-plastic material behaviour at the crack tip the influence of residual stresses along the crack faces due to yielding during crack growth at the crack tip is shown. Examples indicate that the determination of local crack opening stresses and of the local crack opening load is possible. Measurements exactly at the crack tip, however, need further investigation.

\section{REFERENCES}

[1] SPATE 8000 Manual. Ometron Ltd., London.

[2] HARWOOD (N.) and CUMMINGS (W.M.) (editors). - Thermoelastic Stress Analysis. Bristol, Philadelphia, New York, Adam Hilger, 1991, 227 p.

[3] SCHLICHT (O.), KRAPF (K.-G.) and RICHTER (M.) - Rißdetektion mit der Thermoelastischen Spannungsanalyse. Materialprüfung, vol.37, 1995, p. 186-190.

[4] HUB (A.) and WÖLFELI (H.-P.). - Detection and Evaluation of Surface Cracks using the Thermoelastic Stress Analysis. Hallai (C.) and Kulcsar (P.) eds., Proc. 13th World Conf. on Non-Destr. Testing, São Paulo, Oct. 18-23, 1992, p. 715-719.

[5] KANNO (A.) and INOUE (Y.). - Evaluation of a Semielliptical Surface Crack by Application of Thermoelastic Techniques. JSME Int. Joumal, Series I, vol. 33, 1990, p. 44-50.

[6] STANLEY (P.) and DULIEU-SMITH (J.M.). - Progress in the Thermoelastic Evaluation of Mixed-Mode Stress Intensity Factors. Soc. for Exp. Mechanics, SEM Spring Conf. on Exp. Mech., Dearborn, June 7-9, 1993, p. 617-625.

[7] ELBER (W.). - The Significance of Fatigue Crack Closure. Am. Soc. for Testing and Mater. STP 486, 73rd Annual Meeting, Toronto, June 21-26, 1970, p.230-242.

[8] KUMAR (R.) - Review on Crack Closure for Constant Amplitude Loading in Fatigue. Engng. Fract. Mech. vol.42, 1992, p.389-400.

[9] ABAQUS Standard User's Manual. Hibbitt, Karlsson \& Sorensen, Inc., Pawtucket, RI, USA.

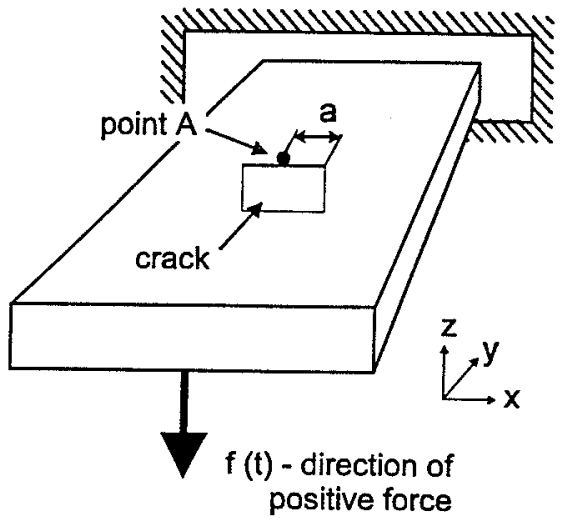

fig.1. Epoxy specimen used for tests

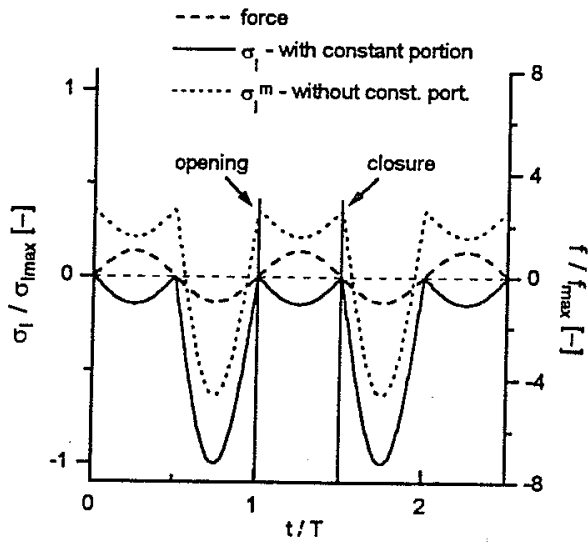

fig.2. Results of calculation for epoxy specimen 


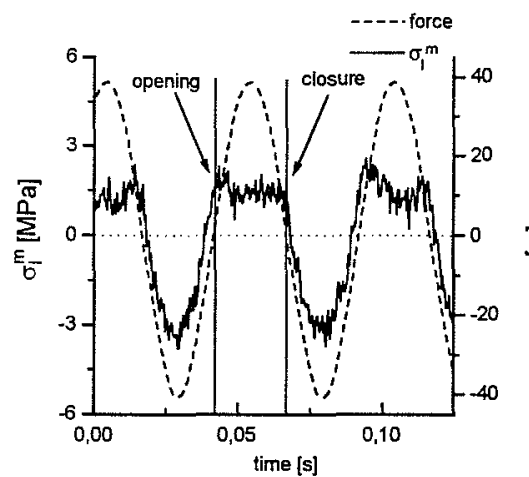

fig.3. Measurement at epoxy specimen (no constant load)

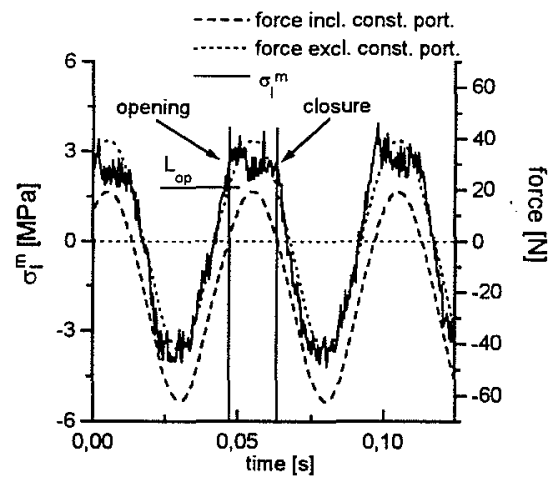

fig.5. Measurement at epoxy specimen (constant compressive load)

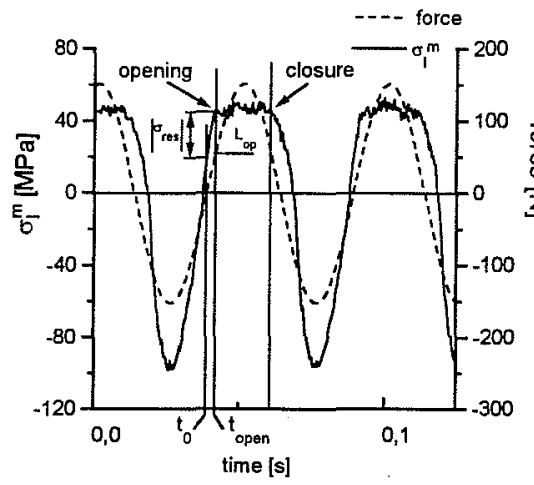

fig.7. Measurement at aluminium specimen with residual compressive stresses

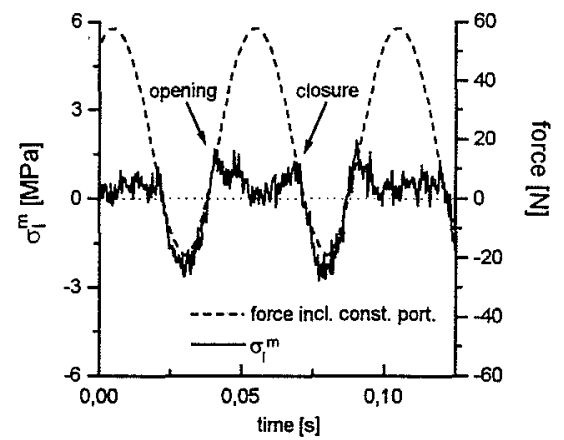

fig.4 . Measurement at epoxy specimen (constant tensile load)

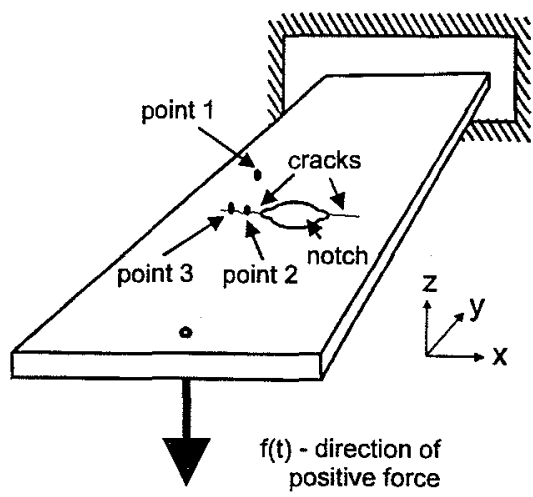

fig.6. Aluminium specimen used for tests

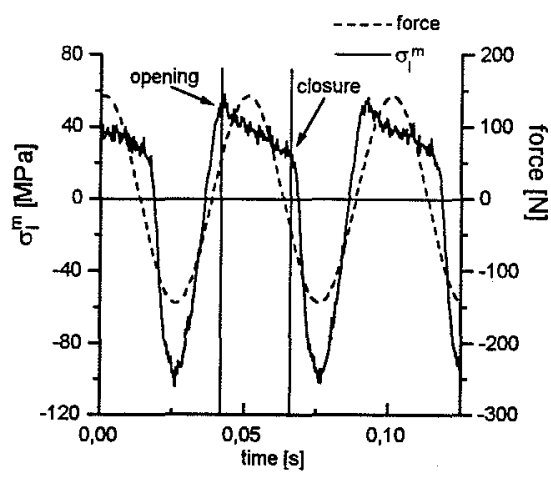

fig.8 . Masurement at aluminium specimen with residual compressive stresses and elastic-plastic behaviour at the crack tip 